\title{
NANOMATCH: A EUROPEAN PROJECT TO DEVELOP CONSOLIDANTS THROUGH THE SYNTHESIS OF NEW INORGANIC NANOMATERIALS FOR THE CONSERVATION OF BUILT HERITAGE
}

\author{
A. Bernardi a , M. Favaro b, T. Nijland c, O. García d, V. Detalle e, K. Wittstadt f, M.D. Romero Sanchez g, L. Pockelé h, B. \\ Kunday i, B. Verhey j, U. Brinkmann k, G. de’ Micheli 1, M. Labouré m, B. Möller n, I.D. Olteanu o \\ a CNR-ISAC, Corso Stati Uniti 4, 35127 Padova, Italy - a.bernardi@isac.cnr.it \\ b CNR-ICIS, Corso Stati Uniti 4, 35127 Padova, Italy - favaro@icis.cnr.it \\ c TNO, The Netherlands - timo.nijland@tno.nl \\ d TECNALIA, C/Geldo - Parque Tecnológico de Bizkaia, 48160 Derio (Bizkaia), Spain - Oihana.garcia@tecnalia.com \\ e Cercle des partenaires du Patrimoine - TRMH, 77420 Champs-sur-Marne, France - vincent.detalle@culture.gouv.fr \\ f Fraunhofer E.V, Bronnbach 28, 97877 Wertheim-Bronnbach, Germany - katrin.wittstadt@isc.fraunhofer.de \\ g AIDICO, Camí de Castella 4, 03660 Novelda- Alicante, Spain - md.romero@aidico.es \\ h R.E.D. srl, Viale dell'industria 58B, 35129 Padova, Italy - luc.pockele@red.srl.com \\ i NANOTEGO INC. GOSB, Teknopark High Tech Binas1 K1 A10, 44184 Gebze Kocaeli, Turkey - burcu.okan@antimic.com \\ j Bofimex B.V, Krombraak 3, 4906 CR Oosterhout state, The Netherlands - bas.verhey@bofimex.nl \\ k Metropolitankapitel der Hohen Domkirche Köln Dombauverwaltung, Roncalliplatz 1, D-50667 Köln, Germany - \\ ulrike.brinkmann@dombau-koeln.de \\ 1 Opera di Santa Croce, Piazza S.Croce 16, 5122 Firenze, Italy - amministrazione@santacroceopera.it \\ m Eschlimann, rue Ettore Bugatti, PO Box 40100,67150 Erstein Cedex, France - laboure@eschlimann.fr \\ n T_O_P Oberflächen GmbH, Friedrichstraße 10a, 97082 Würzburg, Germany - moeller@top-coating.de \\ o SC DUCT SRL, Aviator Stefan Sanatescu 41, 11476 Bucharest, Romania - ductiulian@yahoo.com
}

KEY WORDS: Metal alkoxides, nanostructured materials, consolidants, biocides, built heritage conservation

\begin{abstract}
:
The problem of deterioration of historical building materials, namely stone, wood and glass has become more and more urgent. Climate changes have increased the impact of natural decay whilst socio-economic requirements claim a more sustainable use of existing built heritage. The EU project NANOMATCH addresses this problem through the development of a family of innovative materials. These are specifically designed for the consolidation requirements of historical substrates and for the production of high performance products to fill the gap in the market dedicated to the conservation of built heritage.

Metal-alkoxide precursors will be synthesized and their properties will be tuned based on the substrate characteristics of respectively stone, wood, glass to fulfil specific functionalities. The consolidation effects will be first evaluated through lab experimentation and subsequently the most suitable metal alkoxides will be tested in different European sites to evaluate also the environmental effects on their performance This will lead to a new generation of nano-products specifically tailored for historic materials in a context of climate change, emerging from the most recent and advanced research in the fields of conservation science and nanotechnologies. The development of suitable products for the treatment of historical materials will finally stop the inappropriate use of several commercial products, especially polymers. These have shown in recent years detrimental effects due to their fast deterioration and have also hampered the treated material as well.

Central to the project is the synthesis of molecular precursors, nano-coating deposition and assessment of their conservation properties leading to the production of innovative products for the market of conservation in replacement of unfit traditional ones. The basis for their production and market introduction will be developed within the project.
\end{abstract}

\section{INTRODUCTION}

\subsection{Conservation challenges for built heritage}

In a context of changing environment, the variation of natural and anthropogenic atmospheric parameters, i.e. rain, temperature, pollutants, are worsening the decay of indoor and outdoor monuments. Particular attention must be paid to these new risks (Sabbioni, 2007). Moreover the failure of most organic conservation/restoration treatments and the questionable efficiency of inorganic ones, emphasises the urgent need to develop new or to improve actual products and conservation methodologies as alternatives to conventional ones for Cultural Heritage.
Historic buildings are threatened by a great variety of natural deterioration agents (water, soluble salts and micro-organisms) and pollutants which can affect seriously the materials and their surfaces sometimes preciously painted or decorated. Moreover, the same factors acting on historic substrates will also be impacting on the treatment used. Organic polymers, for example, have demonstrated to be subject to weathering due to $\mathrm{UV} /$ sun radiation, water and salts, which reduce their efficiency and removability (Goudie, 1997; Ghedini, 2006).

From this perspective, it is necessary to take these weathering processes into account and not only design new compatible and resistant materials, but also define the most suitable ageing tests.

\footnotetext{
Corresponding author.
} 


\subsection{State of art on available conservation products}

Many products have been proposed and applied to mitigate the deterioration of building materials. They can be grouped in two main categories: organic and inorganic based products.

Several organic strengthening treatments, mainly consolidation, and materials have been used in order to restore original stone cohesion (Ashurst, 2006). Different synthetic organic polymers, i.e. mainly polyacrylate, polyvinylacetate, epoxy and silicones, have been widely used as consolidants and surface coatings for stone to prevent further deterioration since the last century (Price, 1996). These were not tailored specifically to the application on stone artefacts but seemed to respond to some of the main requirements of restoration treatments: reversibility, durability and chemical inertness.

Although they are still largely used, their conservation efficiency has been drastically reconsidered because they often failed on their promises. Severe alteration and degradation processes (such as photo-oxidative reactions leading to chain scission and/or reticulated structures in acrylic products, or hydrolytic and condensation reactions leading to stiff crosslinked structures in silicon-based products) induced either by environmental conditions or by the substrate itself, cause changes in the physico-chemical properties of those consolidants. In the conservation practice these result in a limited removability of the deteriorated polymers from treated surfaces (Favaro, 2006; Favaro, 2007). They are still in use due to the lack of more performing products notwithstanding the poor affinity with the substrate and/or cracking during shrinkage.

Also for wood there are many different chemical, physical and biological agents altering the lignin/cellulose content, going from a loss of mechanical properties to complete degradation of the wooden structure. Therefore, the investigation on new protective treatments/technologies to enhance stability, durability and service-life of wood-based construction materials is also required. Various approaches with organic compounds have been developed to improve the durability of wood under different environmental conditions. In order to protect wood from damage caused by moisture, it has been treated with waxes, resins (in particular acrylic resins, like for instance Paraloid B72). Often it has also been impregnated with toxic chemicals to prevent fungi or mould colonization (Kucerová, 2010). Net, intensive research is required to develop new environmentally sustainable technologies for improving the durability of wood.

Finally glass conservation was limited for a long time to mending broken pieces with lead and exchanging or referring glasses with degraded paint; later-on natural waxes, resins, animal glues and plaster of Paris were introduced. New perspectives were opened when the progress in the chemical industry offered new materials for gluing, protective coatings or paint layer fixing. However, all of those artificial resins were not specially developed for the application on ancient glass, but mainly for industrial purposes (Newton, 1982).

Since the 1950 's various materials have been proposed and used for the conservation of stained glass, including epoxy resins, acrylates and polyurethanes, araldite and Paraloid B72 (Davison, 1984; Davison, 2003; Bettembourg, 1986; Henau, 1996). The all-round application has some drawbacks in many aspects, and the long-term stability of the treatments is for many materials still questionable (Bertelmann \& Marschner, 1996; Jägers, 2000).

Only in the last years new materials were developed especially devoted to the conservation of stained glass (Roemich, 1992)
(Trademark ORMOCER ${ }^{\circledR}$ or an inorganic polymer, called SZA). For the latter product, systems pilot studies are running, but no commercialization has been achieved yet.

Within the EU CONSTGLASS project, prototypes of innovative product were developed and evolved in a nanoporous glass-phase after sol-gel reaction and condensation (Bellendorf, 2010).

In summary, clearly evident and inappropriate interventions, all over Europe, resulted over the years in:

- cracking, scaling and flaking due to salt deterioration enhanced by superficial water repellent treatments,

- detachment of surface layers due to frost in treated materials, that were not showing frost damage prior to treatment,

- blackening, yellowing or discoloration of treated surfaces of buildings due to environmental and pollutant interaction with the applied products.

The reason for this lays mainly in two facts: the use of polymers conceived for other purposes and the impact of climate change on both historic materials and applied preservation products. Future maintenance, conservation and refurbishment will be facing higher costs due to the failure of polymer treatment or poor efficiency of inorganic product applications.

\section{THE NANOMATCH PROJECT}

The NANOMATCH project (Nano-systems for the conservation of immoveable and moveable polymaterial Cultural Heritage in a changing environment (2011-2014)) is a European Project of the FP7. A group of multidisciplinary experts (chemists, physicists, geologists and biologists) with conservators, restorers, producers and end-users will work together. 15 partners are involved: The Istituto di Scienze dell'Atmosfera e del Clima (Coordinator) and Istituto di Chimica Inorganica e delle Superfici of the Consiglio Nazionale delle Ricerche (IT), Nederlandse Organisatie voor Toegepast Natuurwetenschappelijk Onderzoek (NL), Fundacion TECNALIA Research \& Innovation (S), Cercle des partenaires $\mathrm{du}$ Patrimoine-Laboratoire de recherche des monuments historiques (F), Fraunhofer-Gesellschaft zur Foerderung der Angewandten Forschung E.V (D), AIDICO-Instituto Tecnológico de la Construcción (S) together with the SMEs R.E.D. S.R.L. (IT), NANO TEGO INC. (TR), Bofimex Bouwstoffen B.V. (NL), Eschlimann Atelier de restauration Peinture (F), T_O_P Oberflächen GmbH (D), SC DUCT SRL (RO) and the experimental sites Opera di Santa Croce Firenze (IT) and Metropolitankapitel der Hohen Domkirche Köln Dombauverwaltung (D).

\subsection{Aim of the Project}

The overall NANOMATCH objective is the development of alkaline earth and semimetal alkoxides which evolve to nanostructured conservation products compatible with the main materials used in built heritage like stone and wood -even painted- and glass, ensuring enhanced sustainability, compatibility and efficiency compared to conventional conservation products. 
The fundamental key S\&T objectives of this project are:

* to develop an alkaline earth alkoxide for stone and wood conservation and to prototype an already existing aluminium alkoxide product (developed in EU project CONSTGLASS) for glass consolidation;

* to verify and demonstrate the higher performance of these new products in comparison with conventional and even new innovative ones currently available in the market;

* to establish the most suitable methodology to apply these new products;

* to assess their quality with respect to the environment and human health requirements;

* to prepare more economically attractive products for the market, better meeting needs than current products.

\subsection{The metal alkoxides for consolidation}

Metal-alkoxides have great potential for the development of nano-structured materials, thanks to their high versatility of metal functionalization making them suitable molecular precursors for the preparation of metal-alkoxide solutions or nano-particulate inorganic sols. The final result is a sustainable product tailored to the needs of the material to preserve. This consolidated material would eventually become more resistant, with respect to current organic products, and also to environmental/climatic attacks, which differ from site to site. The consequence of this approach is the availability of more sustainable, specific and well performing consolidants, produced faster and at lower costs.

Particularly alkaline earth alkoxides, calcium and magnesium, precursors of corresponding carbonate, can be considered for stone strengthening as an indispensable and essential preliminary action for its conservation. Furthermore, the same material will be used for wood as these materials, besides strengthening effects, also ensure an alkaline supply to mitigate acidic deterioration processes of cellulose structures. As for wood it is desirable to enhance bioresistance and cohesion. The basic $\mathrm{Ca} / \mathrm{Mg}$ alkoxides will be complemented via known technology with biocide functionalities by adding $\mathrm{Zn} / \mathrm{Cu}, \mathrm{TiO} 2$ nanoparticles or organic molecules. For fractured glass consolidation, the promising aluminium alkoxides precursor of $\mathrm{Al2O} 3$, developed in EU project CONSTGLASS, will be further optimized and prototyped.

Alkaline earth and semimetal alkoxides are in fact suitable molecular precursors of consolidants for stone (Favaro, 2008), wood and glass because the corresponding solutions or nanoparticulate inorganic sols, upon evaporation of the solvent based carrier, undergo hydrolysis and condensation or carbonation when inside the porous structure of the substrate. This was demonstrated for stones and wall paintings in feasibility tests performed in the bilateral Italian-French project GALILEO (Duchêne, 2012) (Figure 1). This results in the deposition of a nano-structured coating on the substrate pore walls (stone, wood) or in internal cracks (glass).

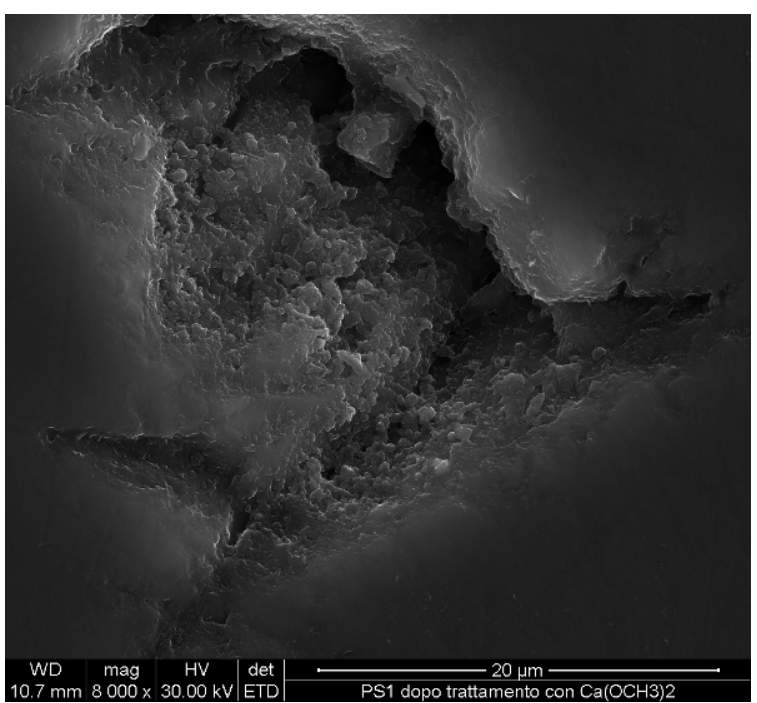

Figure 1: Secondary electron images collected by FEG-ESEM on marble treated with alcoholic solution of Ca-alkoxides. The homogeneous $\mathrm{CaCO} 3$ coating has uniformly adhered on stone pore surfaces.

Besides that, the reacted compounds, carbonates and/or oxides, have the property to adhere well to the original minerals or wood structure of the substrate. By appropriate molecular tuning in function of the substrate requirements, they appear to be stable under major physical, chemical and microbial attacks, restoring integrity and (mechanical) properties of the original matrix.

\subsection{Synthesis of metal alkoxides and Evaluation of their conservation properties}

To reach these objectives the precursors will be designed by selecting appropriate reagents reacting with the alkaline earth metals $\mathrm{Ca}$ and/or $\mathrm{Mg}$ to obtain the desired consolidant for stone and wood. Different synthesis routes will be tested, according to the requirements of the selected final products through modification of organic substituents or through the doping with other metals or biomolecules, to obtain a biocide effect. For the already synthetized Al-alkoxide precursor of the glass consolidant the fundamental step will be the optimization of the sol-gel process deposition. Appropriate application methodologies will be developed and identified in laboratory, also avoiding detrimental effects (in terms of colour or structural changes) on any polychrome that might be on decorated surfaces or wall paintings.

After assessment of the applicability and workability of metalalkoxides solutions, the compatibility of the nanostructured materials with the different substrates and their performance regarding the specific properties to be improved will be tested, and also compared to current commercial and other more recent preservation materials (Ksinopoulou, 2012; MaravelakiKalaitzaki 2012). Tests will be performed according to parameters established previously in the selection of substrates and in the acceptance criteria defined for compatibility and performance assessment together with a durability evaluation. This lab experimentation will identify the boundary condition to perform a suitable conservation treatment taking into account 1 . the effect of damage condition prior and environment condition during the treatment, 2. The influence of the treatment upon pore size distribution, structure, mineralogical and chemical composition of the substrates. Outputs from this work will be 
the identification of suitable products and application methodologies to be tested on site on each substrate.

Field-exposure experiments will be performed on untreated and treated substrate models, eventually previously aged, to evaluate the synergistic interactions of environmental parameters with the substrates and to evaluate the behaviour of the innovative products also in comparison with the commercial ones. During this preliminary one-year survey, the best performing treatment will be tested on small areas of historic substrates to assess the effective interaction of nanomaterials on weathered surfaces. The compatibility effect on treated historic materials will be assessed on the basis of aesthetical, physical, mechanical, chemical issues. The treatment effects will be evaluated on ongoing deterioration processes, on surface appearance, on variation of the hygroscopic behaviour that may differ with the exposure of adjacent untreated materials. An evaluation of risks $\&$ benefits as consequence of the treatment will lead to the definition of damage scenarios and associated risks as future damage development, based upon expert opinions.

As field test sites, four different urban locations in Europe were chosen in accordance with the different climate and environmental conditions of European countries (Figure 2):

- Germany: Cologne Cathedral;

- Italy: Santa Croce Basilica in Florence;

- Spain: Oviedo Cathedral;

- Romania: Stavropoleos Monastery in Bucharest.

All the selected monuments have the three different building material types, i.e. stone, wood and glass. Each of these monuments has particular regional features regarding composition, manufacturing technology and decay pattern. The preservation effects of alkoxides will be evaluated in all sites both on replicas, appropriately identified, and on real surfaces during 18 months to assess the climatological and environmental indoor and outdoor effects on alkoxides performance. At the same time, the same treated samples of previously chosen materials will be exposed in all the climatological regions to compare the effect of the different environments.

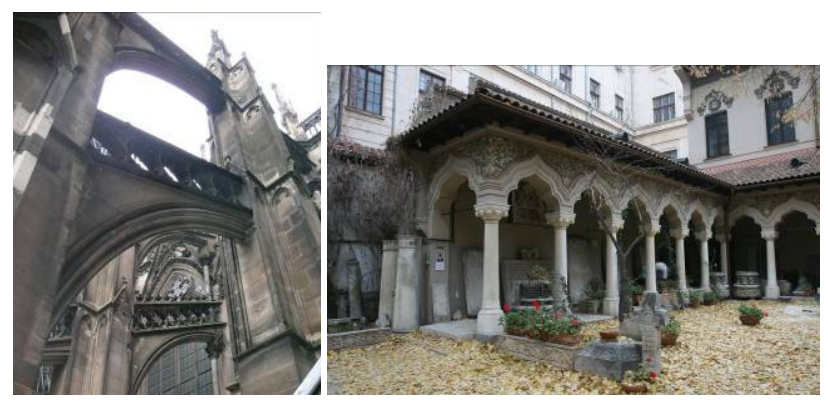

Figure 2. Example of different decay phenomena on outdoor stone materials in two of the selected sites: extensive biocolonization in Cologne Cathedral (Germany) (left) and surface dissolution and erosion on Stavropoleos Monastery in Bucharest (right).

Finally, their eventual safety hazards towards man and environment will be evaluated. Once their performance and safety has been verified, the prototype products will be produced and also the procedure for their introduction in the market will be defined.

\subsection{How the NANOMATCH Project will advance the state of the art}

NANOMATCH project is highly innovative and provides strong advances to the state of art for the following main reasons:

- the final products are new advanced compatible and sustainable nano-structured materials starting from the same class of compounds, tailored in relation to the conservation needs of the different substrates;

- the possibility of tailoring the precursors offers a variety of possible applications in producing products for restoration and protection of different types of stone, wood and glass;

- they are compatible with the substrates because the products have the same chemical and mineralogical composition as the substrate in the case of stone, while for wood and glass they are able to bond to the substrates because of their high chemical affinity;

- appropriate design of the molecular architectures will tune the final properties of the strengthening nano-structures in compliance with the physico-chemical properties of the substrates, without detrimental effect on any polychrome that might be present;

- their common base simplifies the process of the synthesis and the subsequent production of the final products;

- although not reversible, the products fulfil the most important requirements for a restoration product, i.e. compatibility, durability and retreatability;

- thanks to the chosen starting material, they are expected to be very low cost products;

- they are simple in the application methodology;

- an appropriate tailoring of the precursors of metal alkoxides and selection of the additives will tune the final properties of the resulting nanostructured materials to be effective towards the environmental changes.

\section{FUTURE IMPACT}

The NANOMATCH product will mark a new generation of restoration and protecting products, compatible with the original materials, applicable to indoor and outdoor Cultural Heritage.

The basic assumptions of compatibility and/or chemical affinity are at the basis of the expected positive impact in stone and wood applications. NANOMATCH will help to find the best solutions to slow down the damage process and provide tools to mitigate the incoming new mechanisms of deterioration. Thanks to their functionality and versatility they could be useful also in other applications such as tuning surface properties of metals, textiles, ceramics etc., enlarging the possibility of exploitation and market diffusion for the alkoxides derived products.

The new metal alkoxides, based on preliminary costs for production and application, have a promising potential which will be further explored within the project itself.

The starting materials, the precursors, are low cost materials. The synthesizing and subsequent production processes are well known and proven processes. In addition, as the metal-alkoxides constitute a common base for a wide range of substrates and as the fine tuning process is based on simple mixing and matching, the product costs are expected to be well affordable.

In fact, the idea at the base of the project derives from a concept used successfully in industry where basic components, so-called building blocks, are developed and then blended in different ratios to make up several final products adapted to different needs. This approach enlarges the potential use of the 


\section{SYNTHESIS OF NEW INORGANIC NANOMATERIALS FOR THE CONSERVATION OF BUILT HERITAGE}

technology in more markets and applications with economies of scale and cost reductions as consequence since the technological building blocks have to be developed only once. Metal alkoxides with consolidant and biocide products, in NANOMATCH project, constitute such building blocks for the industrial production and market distribution processes.

Since well-known synthesizing, production and application processes will be used like the controlled decomposition and sol-gel technique, the complexity will be of the same order of the existing products. However, due to the long lasting effects and the reduction of retreatability needs, the frequency and extent of application will be lower and hence the total cost.

Finally, as the application covers a wide range of substrates with their varieties, the economies of scale are expected to be higher than for the current products.

The increasing need to share scientific knowledge for the protection and conservation of cultural heritage at the European level is also addressed by the Project, in particular at a time when the environment is changing also as a consequence of political management. The project will promote close collaboration of SME professionals with researchers to steer basic research towards effective application in each development phase: development of suitable synthesis routes which can be translated into production processes, identification of application methodologies which are easy and safe to handle for conservation professionals (architects, restorers, contractors), formulation of compatible and durable innovative materials tuned to historic stone, wood and glass substrates ensuring a long lasting efficiency and reducing future maintenance costs after conservation.

NANOMATCH will enhance the co-operation between SME's, public and private research institutions on national and European scale and the potential benefit of the SME's. The project will create new, highly qualified and attractive jobs in the field of innovative material production and in added value services of historic material consolidation. It has the potential to create also new spin-off initiatives. Also indirect jobs can be created mostly linked to cultural tourism promoting social dynamism and conservation of historic urban areas and finally the quality of life of the citizens living in historic cities can be improved. In fact the visual effect that deterioration of historic surfaces causes in monuments produces strong societal impact. Built heritage deterioration and subsequent inadequate conservation causes heritage damage, insecurity to people living/visiting the affected cities and increases social degradation of cities.

The results obtained in NANOMATCH will certainly be of general interest and could be standardised and utilised by all the European States and not only the involved ones. The whole community interested on the conservation of Cultural Heritage in the world could be interested in this innovation that could strongly change the approach of the conservation methodology and strategy.

\section{CONCLUSIONS}

In conclusion NANOMATCH can provide a "new" category of compatible and sustainable nanostructured inorganic compounds with consolidant, protective and biocide effects. These will strengthen and preserve different damaged or weakened building materials, i.e. stone, wood and glass materials. Furthermore, their performance is expected to last longer than today's solutions available in the market.

The metal-alkoxides have either the same composition or a major chemical affinity and compatibility with the historic substrates in comparison to the products currently available on the market. The possibility to fine tune the product to the different substrates will further enhance the durability. Thanks to these characteristics, these products are inherently lasting longer.

During the project enquiries with SME's, restoration associations in several countries, large monument owners and associations of private owners of historical buildings will be done. This will not only confirm or increase the market potential estimate, but also provide further market insight to fine tune the research and improve the exploitation plans which seem to have a big potential in different scientific, societal and cultural fields.

\section{REFERENCES}

Ashurst, J. (et al.), 2006. Conservation of building and decorative stone. London Butterworth-Heinemann 1998, Henry A. (Ed.): Stone Conservation. Principles and Practice, Donhead Shaftesbury.

Bellendorf, P. (et al.), 2010. Presentation at the final International CONSTGLASS conference "A new glass consolidant - development, testing, and application", $27^{\text {th }}-28^{\text {th }}$ May 2010. http://www.constglass.fraunhofer.de.

Bertelmann \& Marschner, 1996. Alternatives au paraloïd B72 pour la fixation des peintures du verre. In: Grisaille, jaune d'argent, sanguine, émail et peinture à froid: techniques et conservation, Forum pour la conservation et la restauration des vitraux, Liège, France, $19^{\text {th }}-22^{\text {nd }}$ June 1996 , pp. 79-84.

Bettembourg, J. M., 1986. Traitement curatif des vitraux. In: $C V$ Newsletter No 39/40, December 1986, pp. 7-14.

Davison, S.A., 1984. Review of adhesives and consolidants used on glass antiquities. Adhesives and consolidants, Paris Congress, The International Institute for Conservation of Historic and Artistic Works (Ed.), pp. 191-194.

Davison, S., 2003. Conservation and restoration of glass. Second edition, London Butterworth-Heinemann.

Duchêne, S. (et al.), 2012. Nanomaterials for consolidation of marble and wall paintings. In: Proc. of 12th International Congress on the Deterioration and Conservation of Stone, Columbia University, New York, 22-26 October 2012.

Favaro, M. (et al.), 2006. Polym. Degrad. Stab. ,91, pp. 30833096.

Favaro, M. (et al.), 2007. Polym. Degrad. Stab. , 92 , pp. 335351.

Favaro, M. (et al.), 2008. Applied Organometallic Chemistry, 22, pp. 698-704.

Ghedini, N. (et al.), 2006. Environmental Science and Technology, 40, pp. 939-944.

Goudie, A. (et al.), 1997. Salt weathering hazards. Wiley, Chichester.

Henau, P. 1996. Le paraloïd B72 pour fixer la grisaille et l'émail. In: Grisaille, jaune d'argent, sanguine, émail et peinture à froid: techniques et conservation, Forum pour la conservation et la restauration des vitraux, Liège, France, June 19-22 1996, pp. 139-142. 
Jägers, E. (et al.), 2000. Konservierungsmaterialien und Methoden. In: Restaurierung und Konservierung historischer Glasmalereien, Wolff A. (Ed.), Verlag Philipp von Zabern, Mainz, pp. 129-166.

Ksinopoulou (et al.), 2012 The effectiveness of nanoparticles in the consolidation of porous calcareous stones 4th International Symposium on Nanotechnology in Construction, Agios Nikolaos, Greece, May 20-22, 2012

Kucerová, I. (et al.), 2010. Examination of Damaged Wood by Ammonium Phosphate and Sulphate-Based Fire Retardants: The Results of the Prague Castle Roof Timber Examination. In Wood Science for Conservation of Cultural Heritage, Proceedings of the International Conference Hld by Cost Action IE0601 in Florence (Italy), $8^{\text {th }}-10^{\text {th }}$ November 2007 (DOI: $10.1400 / 141804)$

Maravelaki-Kalaitzaki P. (et al.), 2012. Hybrid TEOS/colloidalsilica/TiO2/GPTMS Composites for stone consolidation $4^{\text {th }}$ International Symposium on Nanotechnology in Construction, Agios Nikolaos, Greece, May 20-22, 2012

Newton, R. G., 1982. The deterioration and conservation of painted glass - a critical bibliography. Oxford University Press, Oxford, UK.

Price C. A., 1996. Stone Conservation: An Overview of Current Research. J. Paul Getty Trust, Los Angeles, CA.

Roemich, H.D. (et al.), 1992. Bol. Soc. Esp. Ceram., 31-C, pp. 137-141.

Sabbioni, C. (et al.), 2007. Global Climate Change Impact on Built Heritage and Cultural Landscape. The Noah's Ark Project, Atlas and Guidelines (ISBN 978889030280 0).

\section{ACKNOWLEDGEMENTS}

The European Commission is kindly acknowledged for the financial support to NANOMATCH project (FP7/2007-2013: grant agreement no. 283182, Nano-system for the conservation of immovable and movable polymaterial Cultural Heritage in a changing environment, Coordinator: Dr. Adriana Bernardi, National Research Council - Institute of Atmospheric Science and Climate). 